\title{
Charlier Series Solutions of Systems of First Order Delay Differential Equations with Proportional and Constant Arguments
}

\author{
Ömür Kıvanç Kürkçü1,* $\odot$, Mehmet Sezer²® \\ ${ }^{1}$ Department of Engineering Basic Sciences, Konya Technical University, Konya, Turkey \\ 2 Department of Mathematics, Manisa Celal Bayar University, Manisa, Turkey \\ *Corresponding author: omurkivanc@outlook.com
}

Received: 31.10.2021 Accepted: 27.01.2022

\begin{abstract}
This study is devoted to obtaining the Charlier series solutions of first order delay differential equations involving proportional and constant arguments by employing an inventive numerical method dependent upon a collaboration of matrix structures derived from the parametric Charlier polynomial. The method essentially conducts the conversion of the unknown terms into a unique matrix equation at the collocation points, which yields a direct computation for these stiff equations. Two illustrative examples are included to test the accuracy and efficiency of the method. According to the investigation of the graphical and numerical results, the method holds fast, inventive and accurate computation, regularizing the matrix forms in compliance with the equations in question.
\end{abstract}

Keywords: Charlier polynomial, Collocation points, Delay arguments, Matrix method

\section{Introduction}

In this paper, the main aim is focused on obtaining the Charlier series solutions of the systems of the delay differential equations involving proportional and constant arguments (SDDEs) in the form

$$
\sum_{k=0}^{1} \sum_{j=1}^{2}\left(P_{i j}^{k}(x) y_{j}^{(k)}(x)+Q_{i j}^{k}(x) y_{j}^{(k)}\left(\alpha_{j k} x+\beta_{j k}\right)\right)=g_{i}(x), \quad i=1,2
$$

on $[\mathrm{a}, \mathrm{b}]$, subject to the initial conditions $y_{i}(a)=\lambda_{i}$. Here, $P_{i j}^{k}(x), Q_{i j}^{k}(x)$ and $g_{i}(x)$ are continuous functions; $\alpha_{j k}$ and $\beta_{j k}$ are proportional and constant delay arguments $\left(\alpha_{j k} \in(0,1], \beta_{j k} \in[-1,1] /\{0\}\right)$, respectively. The Charlier series solutions are extracted by the parametric Charlier polynomial, as

$$
y_{i}(x) \cong \sum_{n=0}^{N} a_{i n} C_{n}(x, \alpha), \alpha>0,
$$

where $a_{i n}{ }^{\prime}$ s are unknown Charlier coefficients to be released by the method and $C_{n}(x, \alpha)$ implies the Charlier polynomials with the parameter $\alpha$. 
As a special form of SDEs, the systems of differential equations with time delays (SDDEs) are of great importance in the modelling of population dynamics, such as predator-prey systems, and HIV infection of CD4+ T-cells (Gokmen et al., 2015; Culshaw and Ruan, 2000; Kuang, 1993; Cushing, 1977). Recently, these type systems also continue to undergo the sustainable development for specific models, such as drug therapy for HIV infection via the system of fractional differential equations (Thirumalai et al., 2021). It is acceptable to express that the analytical solutions of SDEs are barely obtained via any analytical procedure. Thus, some efficient numerical methods are prepared to consider this issue. So far, a Chebyshev polynomial method has been proposed for the systems of higher order differential equations (Akyüz and Sezer, 2003). The Taylor collocation method has been used for linear differentialdifference equations (Gökmen and Sezer, 2013). He's variational iteration method has been employed for solving the systems of differential equations (Tatari and Dehghan, 2009). The exponential Galerkin method and the optimal perturbation iteration method have been proposed for treating a HIV infection model of CD4+ T-cells (Yüzbaşı and Karaçayır, 2017; Deniz, 2021). The optimal perturbation iteration method has also been applied to approach to the solution of a fractional Ebola virus disease model (Srivastava and Deniz, 2021). The Adomian decomposition method has been utilized to solve the continuous population models for single and interacting species (Pamuk, 2005). The differential transformation method has been utilized to obtain the solutions of systems of differential equations (Abdel-Halim Hassan, 2008).

Having been motivated by the studies above, this study deals with an inventive numerical method based on the Charlier polynomials to readily solve SDDEs, collaborating the matrix expansions with regard to the terms in Eq. (1).

This study is organized as follows: Section 2 reveals some properties of the Charlier polynomials. Section 3 comprises the method of solution via the collaboration of the matrices. Section 4 processes two numerical examples to exhibit the accuracy and efficiency of the present method. Section 5 discusses the innovations and outcomes, which are produced by the method presented in Section 4.

\section{Some properties of Charlier polynomials}

The discrete Charlier polynomials can be given by the generating function, as (Dunster, 2001; Szegö, 1975)

$$
\sum_{n=0}^{\infty} C_{n}(x, \alpha) \frac{z^{n}}{n !}=\frac{(1+z)^{x}}{e^{\alpha z}},
$$

and their explicit formulation can also be of the form (Dunster, 2001; Szegö, 1975)

$$
C_{n}(x, \alpha)=\sum_{k=0}^{n} k !\left(\begin{array}{l}
n \\
k
\end{array}\right)\left(\begin{array}{l}
x \\
k
\end{array}\right)(-\alpha)^{n-k} .
$$

They govern a discrete orthogonality relation stemmed from the Poisson distribution (Roman, 1984; Dunster, 2001)

$$
\sum_{k=0}^{\infty} C_{m}(k, \alpha) C_{n}(k, \alpha) w(k)=\alpha^{n} n ! \delta_{m n}, \alpha>0
$$

subject to the Poisson density $w(k)=\alpha^{k} /\left(e^{\alpha} k !\right)$ in terms of the parameter $\alpha$. 
These polynomials possess a recurrence relation (Dunster, 2001)

$$
C_{n+1}(x, \alpha)=(x-n-\alpha) C_{n}(x, \alpha)-\alpha n C_{n-1}(x, \alpha) .
$$

On the other hand, the uniform asymptotic expansions of the Charlier polynomials were discussed in (Dunster, 2001). The uniform and non-uniform asymptotics of the Charlier polynomials were studied using difference equation methods in (Huang et al., 2021). The Szász type operators composed of the Charlier polynomials were derived in (Varma and Taşdelen, 2012).

\section{Method of solution: A collaboration of matrices}

In this section, the method is established via the matrices of the terms in Eq. (1) and their collaboration structure via the Charlier polynomials. First, by the Charlier series solution form (2), its matrix relation turns out to be

$$
y_{i}(x)=\boldsymbol{C}(x, \alpha) \boldsymbol{A}_{i}=\chi(x) \boldsymbol{L}(\alpha) \boldsymbol{A}_{i}, i=1,2,
$$

where

$$
\begin{gathered}
\boldsymbol{C}(x, \alpha)=\left[\begin{array}{llll}
C_{0}(x, \alpha) & C_{1}(x, \alpha) & \cdots & C_{N}(x, \alpha)
\end{array}\right], \chi(x)=\left[\left(\begin{array}{l}
x \\
0
\end{array}\right)\left(\begin{array}{l}
x \\
1
\end{array}\right) \cdots\left(\begin{array}{c}
x \\
N
\end{array}\right)\right], \\
\boldsymbol{A}_{i}=\left[\begin{array}{llll}
a_{i 0} & a_{i 1} & \cdots & a_{i N}
\end{array}\right]^{T},
\end{gathered}
$$

and

$$
\boldsymbol{L}^{T}(\alpha)=\left[\begin{array}{ccccc}
l_{00}(\alpha) & 0 & 0 & \cdots & 0 \\
l_{10}(\alpha) & l_{11}(\alpha) & 0 & \cdots & 0 \\
l_{20}(\alpha) & l_{21}(\alpha) & l_{22}(\alpha) & \cdots & 0 \\
\vdots & \vdots & \vdots & \ddots & \vdots \\
l_{N 0}(\alpha) & l_{N 1}(\alpha) & l_{N 2}(\alpha) & \cdots & l_{N N}(\alpha)
\end{array}\right]
$$

such that

$$
l_{m n}(\alpha)=\left\{\begin{array}{cc}
n !\left(\begin{array}{c}
m \\
n
\end{array}\right)(-\alpha)^{m-n}, & m \geq n \\
0 & m<n
\end{array}, m, n=0,1, \ldots, N .\right.
$$

It is obvious that the matrix relation (3) is not ready to be involved in the method since it is composed of non-smooth polynomial expansion. An inventive transitive matrix relation is thus, deployed as follows:

Theorem 3.1. Let $s(n, k), S_{1}$ and $\boldsymbol{X}(x)$ be the Stirling number of the first kind, a upper triangular matrix and a Taylor polynomial base, respectively. Then, a transitive matrix relation is constructed as

$$
\chi(x)=\boldsymbol{X}(x) \boldsymbol{S}_{1}
$$

where 


$$
\boldsymbol{X}(x)=\left[\begin{array}{llll}
1 & x & \cdots & x^{N}
\end{array}\right] \text { and } S_{1}=\left[\begin{array}{ccccc}
\frac{s(0,0)}{0 !} & \frac{s(1,0)}{1 !} & \frac{s(2,0)}{2 !} & \cdots & \frac{s(N, 0)}{N !} \\
0 & \frac{s(1,1)}{1 !} & \frac{s(2,1)}{2 !} & \cdots & \frac{s(N, 1)}{N !} \\
0 & 0 & \frac{s(2,2)}{2 !} & \cdots & \frac{s(N, 2)}{N !} \\
\vdots & \vdots & \vdots & \ddots & \vdots \\
0 & 0 & 0 & \cdots & \frac{s(N, N)}{N !}
\end{array}\right] .
$$

Proof. By making use of a combinatorial property, which is presented in (Butzer et al., 1989), as

$$
\left(\begin{array}{l}
x \\
n
\end{array}\right)=\sum_{k=0}^{n} \frac{s(n, k) x^{k}}{n !}
$$

it can be expanded via the matrix relations, as

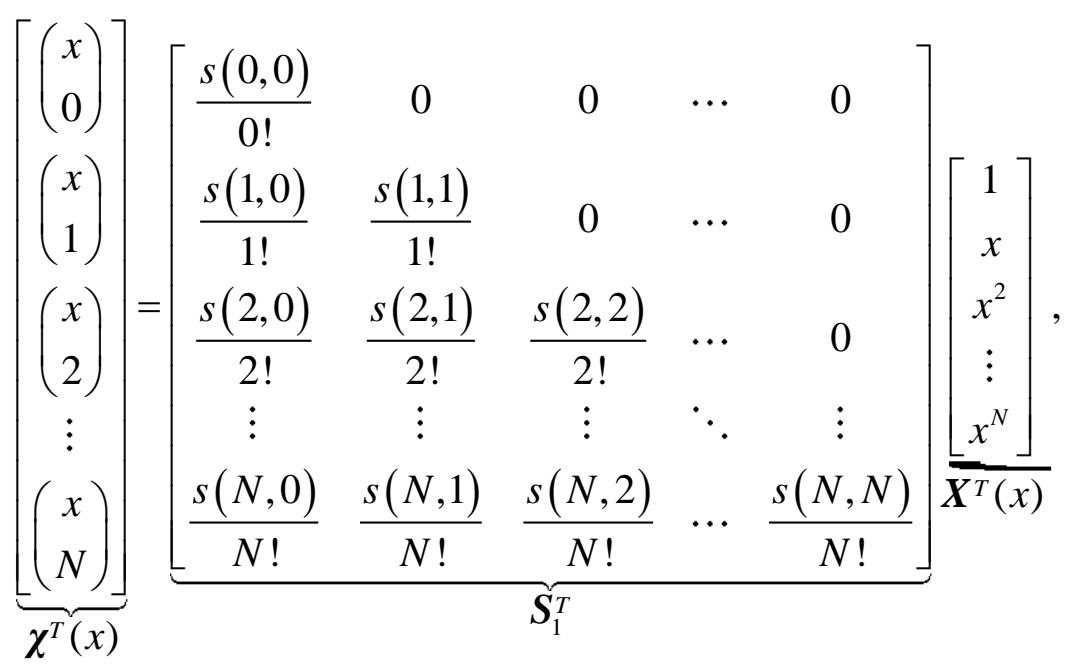

hence, a transitive matrix relation can be finalized as

$$
\boldsymbol{\chi}^{T}(x)=\boldsymbol{S}_{1}^{T} \boldsymbol{X}^{T}(x) \Rightarrow \chi(x)=\boldsymbol{X}(x) \boldsymbol{S}_{1},
$$

which completes the proof.

Now, after inserting the transitive matrix relation (4) into the matrix relation (3), the matrix relation of the solution form leads to

$$
y_{i}(x)=\boldsymbol{X}(x) \boldsymbol{S}_{1} \boldsymbol{L}(\alpha) \boldsymbol{A}_{i}=\boldsymbol{X}(x) \boldsymbol{M}(\alpha) \boldsymbol{A}_{i} ; \boldsymbol{M}(\alpha)=\boldsymbol{S}_{1} \boldsymbol{L}(\alpha), i=1,2 .
$$

To deploy the matrix relation (5) in Eq. (1), taking its differentiation of $k$-th order, we have

$$
y_{i}^{(k)}(x)=\boldsymbol{X}(x) \boldsymbol{T}^{k} \boldsymbol{M}(\alpha) \boldsymbol{A}_{i},
$$

where 


$$
\boldsymbol{T}^{k}=\left[\begin{array}{ccccc}
0 & 1 & 0 & \cdots & 0 \\
0 & 0 & 2 & \cdots & 0 \\
\vdots & \vdots & \vdots & \ddots & \vdots \\
0 & 0 & 0 & 0 & N \\
0 & 0 & 0 & 0 & 0
\end{array}\right]^{k}
$$

On the other hand, the matrix relation of the term with delay arguments in Eq. (1) can be expanded after substituting $x \rightarrow \alpha_{j k} x+\beta_{j k}$ into the matrix relation (5). So, we have

$$
y_{i}^{(k)}\left(\alpha_{j k} x+\beta_{j k}\right)=\boldsymbol{X}(x) \boldsymbol{B}\left(\alpha_{j k}, \beta_{j k}\right) \boldsymbol{T}^{k} \boldsymbol{M}(\alpha) \boldsymbol{A}_{i}, i=1,2,
$$

where $\boldsymbol{B}\left(\alpha_{j k}, \beta_{j k}\right)$ is the binomial coefficient matrix obtained from the expansion of $\left(\alpha_{j k} x+\beta_{j k}\right)^{N}$ and is of the form (see (Gökmen and Sezer, 2013) for its earlier form)

$$
\boldsymbol{B}\left(\alpha_{j k}, \beta_{j k}\right)=\left[\begin{array}{ccccc}
\left(\begin{array}{l}
0 \\
0
\end{array}\right) \alpha_{j k}^{0} \beta_{j k}^{0} & \left(\begin{array}{l}
1 \\
0
\end{array}\right) \alpha_{j k}^{0} \beta_{j k}^{1} & \left(\begin{array}{l}
2 \\
0
\end{array}\right) \alpha_{j k}^{0} \beta_{j k}^{2} & \cdots & \left(\begin{array}{c}
N \\
0
\end{array}\right) \alpha_{j k}^{0} \beta_{j k}^{N} \\
0 & \left(\begin{array}{l}
1 \\
1
\end{array}\right) \alpha_{j k}^{1} \beta_{j k}^{0} & \left(\begin{array}{l}
2 \\
1
\end{array}\right) \alpha_{j k}^{1} \beta_{j k}^{1} & \cdots & \left(\begin{array}{c}
N \\
1
\end{array}\right) \alpha_{j k}^{1} \beta_{j k}^{N-1} \\
0 & 0 & \left(\begin{array}{l}
2 \\
2
\end{array}\right) \alpha_{j k}^{2} \beta_{j k}^{0} & \cdots & \left(\begin{array}{c}
N \\
2
\end{array}\right) \alpha_{j k}^{2} \beta_{j k}^{N-2} \\
\vdots & \vdots & \vdots & \ddots & \vdots \\
0 & 0 & 0 & 0 & \left(\begin{array}{c}
N \\
N
\end{array}\right) \alpha_{j k}^{N} \beta_{j k}^{0}
\end{array}\right] .
$$

Using the collaboration of the matrix relations (5)-(7), the matrix form of Eq. (1) can be written as

$$
\sum_{k=0}^{1}\left(\boldsymbol{P}_{k}(x) \overline{\boldsymbol{X}}(x)+\boldsymbol{Q}_{k}(x) \overline{\boldsymbol{X}}(x) \overline{\boldsymbol{B}}\left(\alpha_{k}, \boldsymbol{\beta}_{k}\right)\right) \overline{\boldsymbol{T}^{k}} \overline{\boldsymbol{M}}(\alpha) \boldsymbol{A}=\boldsymbol{G}(x),
$$

where

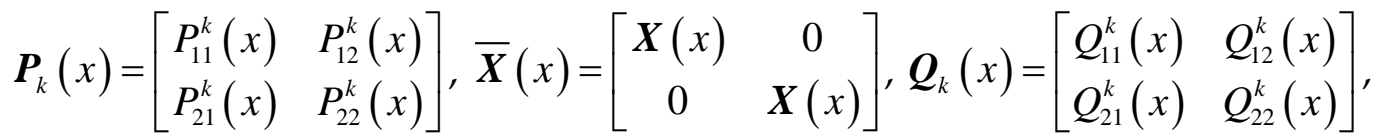

$$
\begin{aligned}
& \overline{\boldsymbol{B}}\left(\alpha_{k}, \beta_{k}\right)=\left[\begin{array}{cc}
\boldsymbol{B}\left(\alpha_{1 k}, \beta_{1 k}\right) & 0 \\
0 & \boldsymbol{B}\left(\alpha_{2 k}, \beta_{2 k}\right)
\end{array}\right], \overline{\boldsymbol{T}^{k}}=\left[\begin{array}{cc}
\boldsymbol{T}^{k} & 0 \\
0 & \boldsymbol{T}^{k}
\end{array}\right], \overline{\boldsymbol{M}}(\alpha)=\left[\begin{array}{cc}
\boldsymbol{M}(\alpha) & 0 \\
0 & \boldsymbol{M}(\alpha)
\end{array}\right], \\
& \boldsymbol{G}(x)=\left[\begin{array}{l}
g_{1}(x) \\
g_{2}(x)
\end{array}\right], \boldsymbol{A}=\left[\begin{array}{l}
\boldsymbol{A}_{1} \\
\boldsymbol{A}_{2}
\end{array}\right]=\left[\begin{array}{llllllll}
a_{10} & a_{11} & \cdots & a_{1 N} & a_{20} & a_{21} & \cdots & a_{2 N}
\end{array}\right]^{T},
\end{aligned}
$$

and with the collocation points $x_{i}=(b-a) i / N$, it admits the fundamental matrix equation

$$
\boldsymbol{W}(\alpha) \boldsymbol{A}=\boldsymbol{G},
$$

where 


$$
\boldsymbol{W}(\alpha)=\sum_{k=0}^{1}\left(\boldsymbol{P}_{k} \overline{\boldsymbol{X}}+\boldsymbol{Q}_{k} \overline{\boldsymbol{X}} \overline{\boldsymbol{B}}\left(\alpha_{k}, \beta_{k}\right)\right) \overline{\boldsymbol{T}^{k}} \overline{\boldsymbol{M}}(\alpha)
$$

such that

$$
\boldsymbol{P}_{k}=\operatorname{diag}\left[\boldsymbol{P}_{k}\left(x_{i}\right)\right], \overline{\boldsymbol{X}}=\left[\begin{array}{llll}
\overline{\boldsymbol{X}}\left(x_{0}\right) & \overline{\boldsymbol{X}}\left(x_{1}\right) & \cdots & \overline{\boldsymbol{X}}\left(x_{N}\right)
\end{array}\right]^{T}, \boldsymbol{Q}_{k}=\operatorname{diag}\left[\boldsymbol{Q}_{k}\left(x_{i}\right)\right],
$$

and

$$
\boldsymbol{G}=\left[\begin{array}{llll}
\boldsymbol{G}\left(x_{0}\right) & \boldsymbol{G}\left(x_{1}\right) & \cdots & \boldsymbol{G}\left(x_{N}\right)
\end{array}\right]^{T} .
$$

In addition, in view of the matrix relation (5), the matrix forms of the initial conditions of Eq. (1) are established as

$$
\left.\begin{array}{l}
y_{1}(a)=\boldsymbol{X}(a) \boldsymbol{M}(\alpha) \boldsymbol{A}_{1}=\lambda_{1} \\
y_{2}(a)=\boldsymbol{X}(a) \boldsymbol{M}(\alpha) \boldsymbol{A}_{2}=\lambda_{2}
\end{array}\right\} \Rightarrow\left[\begin{array}{lll}
\boldsymbol{U}_{1}(\alpha) & : & \lambda_{1} \\
\boldsymbol{U}_{2}(\alpha) & : & \lambda_{2}
\end{array}\right] .
$$

Now, the conditional row matrices (9) are replaced by the proper rows related to $A_{1}$ and $A_{2}$ in Eq. (8). Then, the augmented matrix system is obtained as

$$
\boldsymbol{W}(\alpha) \boldsymbol{A}=\boldsymbol{G} \Rightarrow[\boldsymbol{W}(\alpha): \boldsymbol{G}] .
$$

The matrix system (10) is solved after the parameter $\alpha$ is determined numerically. So, the unknown Charlier coefficients is thus, obtained and as a final step, the Charlier series solution is constructed when these coefficients are substituted into $\boldsymbol{A}_{i}$.

\section{Numerical Examples}

In this section, two illustrative examples are included to test the accuracy and efficiency of the method. In that case, a computer program on Mathematica 11.3 is developed in accordance with the mainframe of the method. Therefore, the computation results of the examples are directly overviewed and compared in tables and figures.

Example 4.1. Consider a system of first order delay differential equations

$$
\left.\begin{array}{l}
y_{1}^{\prime}(x)-x e^{x-1} y_{2}(x-1)=e^{x}-x \\
y_{2}^{\prime}(x)+2 x^{2} e^{-x-1} y_{1}(x+1)=2 x^{2}-e^{-x}
\end{array}\right\}, 0 \leq x \leq 1,
$$

subject to its initial conditions $y_{1}(0)=y_{2}(0)=1$. Here, the exact solutions of the system are of the form $y_{1}(x)=e^{x}$ and $y_{2}(x)=e^{-x}$. This system is solved, by the time the present method is deployed for different $N$. Thus, the Charlier series solutions are demonstrated in Figure 1, where these solutions are in good agreement with the corresponding exact solutions. This consistency can be noticed via different $N$ and the parameter $\alpha$ in Tables 1 and 2, taking approximately eleven decimal places of accuracy. In addition, Tables 1 and 2 reveal that the CPU timing cost of all computations remains low amount in terms of both $N=4$ and $N=10$. 


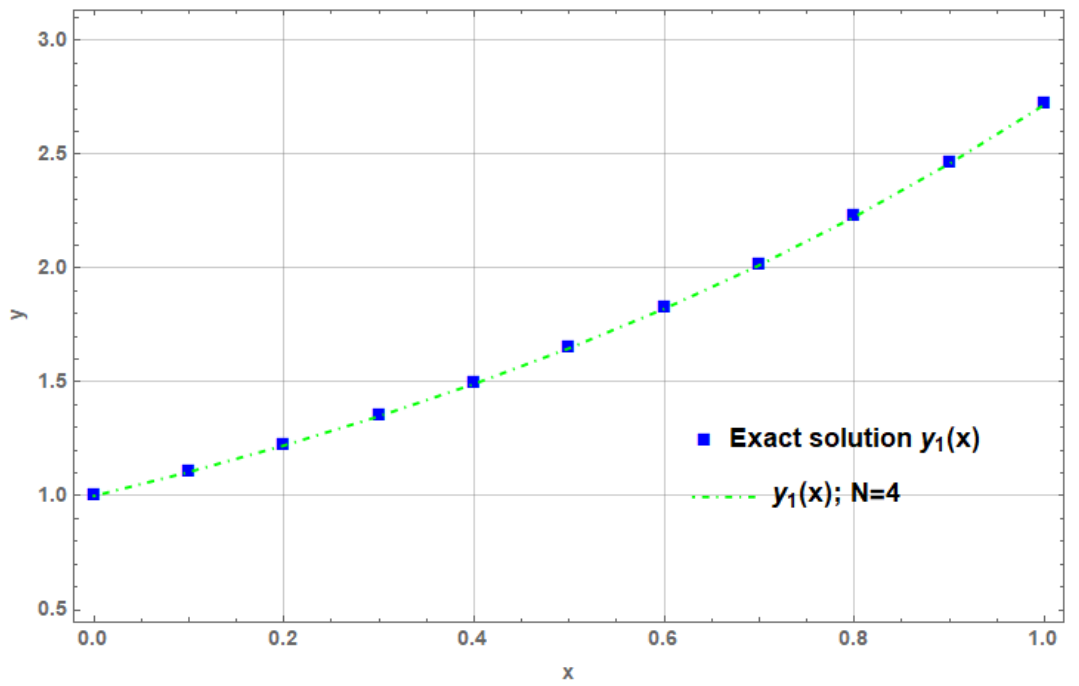

(a) Exact solution and Charlier series solution $y_{1}(x)$ for $\alpha=0.1$

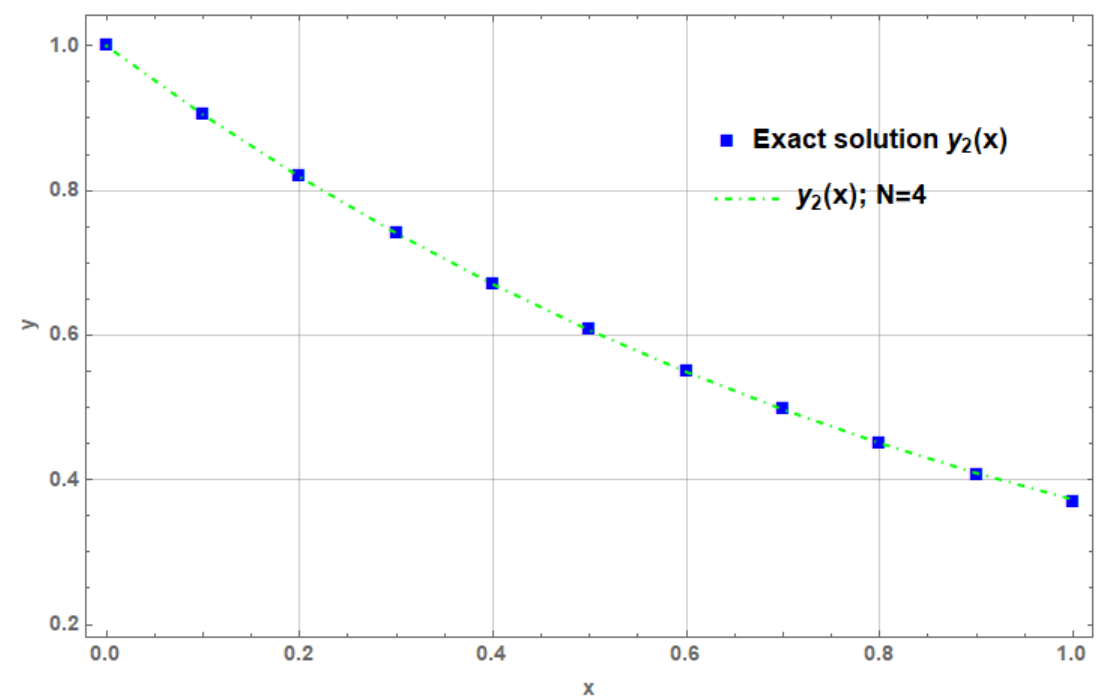

(b) Exact solution and Charlier series solution $y_{2}(x)$ for $\alpha=0.1$

Figure 1. Displacements of the solutions for Example 4.1

Table 1. Comparison of CPU timing and the absolute errors with respect to the Charlier series solutions $\left\{y_{1}(x), y_{2}(x)\right\}$ with $\alpha=0.1$ and $N$ for Example 4.1

\begin{tabular}{lllll}
\hline$x_{i}$ & $\left|e_{1}\left(x_{i}\right)\right|$ & $\left|e_{2}\left(x_{i}\right)\right|$ & $\left|e_{1}\left(x_{i}\right)\right|$ & $\left|e_{2}\left(x_{i}\right)\right|$ \\
& $N=4$ & $N=4$ & $N=10$ & $N=10$ \\
\hline 0.2 & $6.3298 \mathrm{e}-05$ & $1.0146 \mathrm{e}-05$ & $2.6489 \mathrm{e}-011$ & $2.5502 \mathrm{e}-013$ \\
0.4 & $3.2572 \mathrm{e}-05$ & $3.3047 \mathrm{e}-05$ & $6.9767 \mathrm{e}-011$ & $2.3063 \mathrm{e}-011$ \\
0.6 & $2.5548 \mathrm{e}-04$ & $2.7693 \mathrm{e}-04$ & $8.9304 \mathrm{e}-011$ & $7.3378 \mathrm{e}-010$ \\
0.8 & $3.1847 \mathrm{e}-04$ & $1.4273 \mathrm{e}-03$ & $9.2578 \mathrm{e}-011$ & $1.0343 \mathrm{e}-008$ \\
1.0 & $2.1181 \mathrm{e}-04$ & $4.8428 \mathrm{e}-03$ & $9.2488 \mathrm{e}-011$ & $8.7615 \mathrm{e}-008$ \\
\hline Time & & 0.0469 & & 0.5313 \\
\hline
\end{tabular}


Table 2. Comparison of CPU timing and the absolute errors with respect to the Charlier series solutions $\left\{y_{1}(x), y_{2}(x)\right\}$ with $\alpha=1$ and $N$ for Example 4.1

\begin{tabular}{lllll}
\hline \multirow{2}{*}{$x_{i}$} & $\left|e_{1}\left(x_{i}\right)\right|$ & $\left|e_{2}\left(x_{i}\right)\right|$ & $\left|e_{1}\left(x_{i}\right)\right|$ & $\left|e_{2}\left(x_{i}\right)\right|$ \\
& $N=4$ & $N=4$ & $N=10$ & $N=10$ \\
\hline 0.2 & $6.3298 \mathrm{e}-05$ & $1.0146 \mathrm{e}-05$ & $3.7095 \mathrm{e}-011$ & $2.9066 \mathrm{e}-013$ \\
0.4 & $3.2572 \mathrm{e}-05$ & $3.3047 \mathrm{e}-05$ & $8.8780 \mathrm{e}-011$ & $2.3332 \mathrm{e}-011$ \\
0.6 & $2.5548 \mathrm{e}-04$ & $2.7693 \mathrm{e}-04$ & $1.1056 \mathrm{e}-010$ & $7.3501 \mathrm{e}-010$ \\
0.8 & $3.1847 \mathrm{e}-04$ & $1.4273 \mathrm{e}-03$ & $1.1412 \mathrm{e}-010$ & $1.0350 \mathrm{e}-008$ \\
1.0 & $2.1181 \mathrm{e}-04$ & $4.8428 \mathrm{e}-03$ & $1.1405 \mathrm{e}-010$ & $8.7658 \mathrm{e}-008$ \\
\hline Time & & 0.0313 & & 0.4219 \\
\hline
\end{tabular}

\section{Example 4.2. Consider a system of first order delay differential equations}

$$
\left.\begin{array}{l}
y_{1}^{\prime}(x)+x^{2} y_{2}^{\prime}(0.5 x-0.1)+x y_{1}(x)=e^{x}+e^{x} x+x^{2} \cos (0.5 x-0.1) \\
y_{2}^{\prime}(x)-\cos (x) y_{1}^{\prime}(0.5 x+0.5)+y_{2}(x)=\cos (x)-e^{0.5 x+0.5} \cos (x)+\sin (x)
\end{array}\right\},-1 \leq x \leq b,
$$

subject to its initial conditions $y_{1}(-1)=\exp (-1)$ and $y_{2}(-1)=-\sin (1)$. Here, the exact solutions of this system are of the form $y_{1}(x)=e^{x}$ and $y_{2}(x)=\sin (x)$. After solving this system with the aid of the present method for $b=\{1,3\}$, the Charlier series solutions are illustrated in Figure 2, respectively. In addition, these solutions can be simulated on both normal $(b=1)$ and long spatial $(b=3)$ intervals, as in Figures 2 and 3, respectively. The clear approaches to the exact solutions on both figures are determined. Table 3 approves these precisions in numerical aspect and demonstrates the low CPU timing results even for higher computation limit.

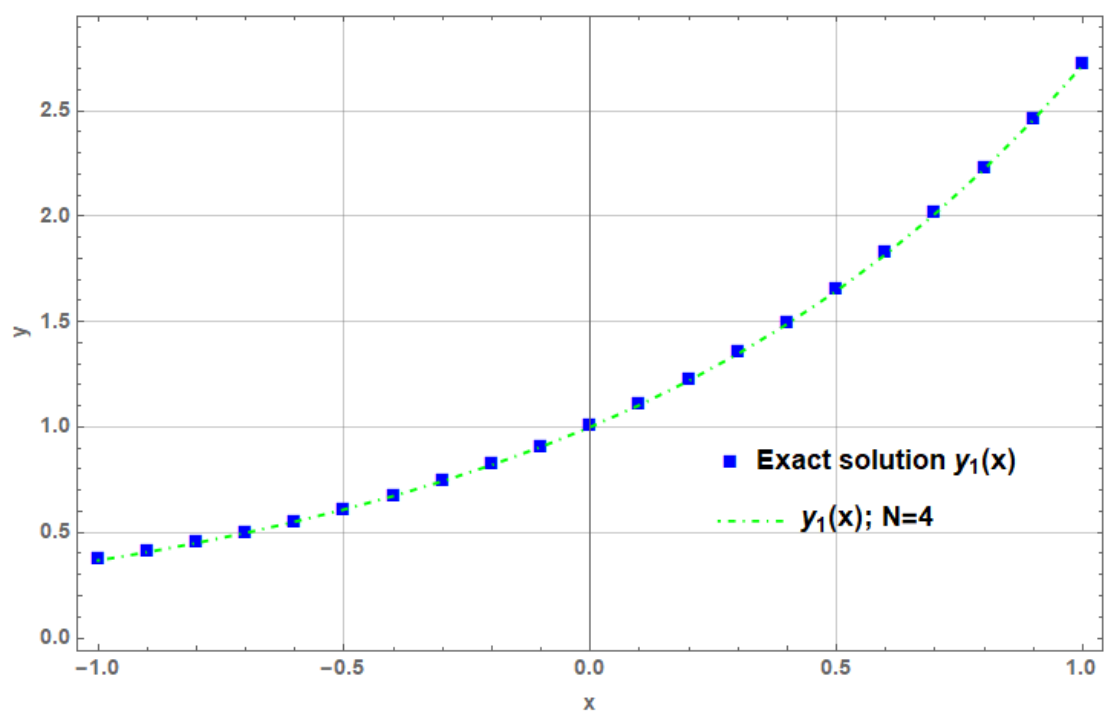

(a) Exact solution and Charlier series solution $y_{1}(x)$ for $\alpha=0.1$ 


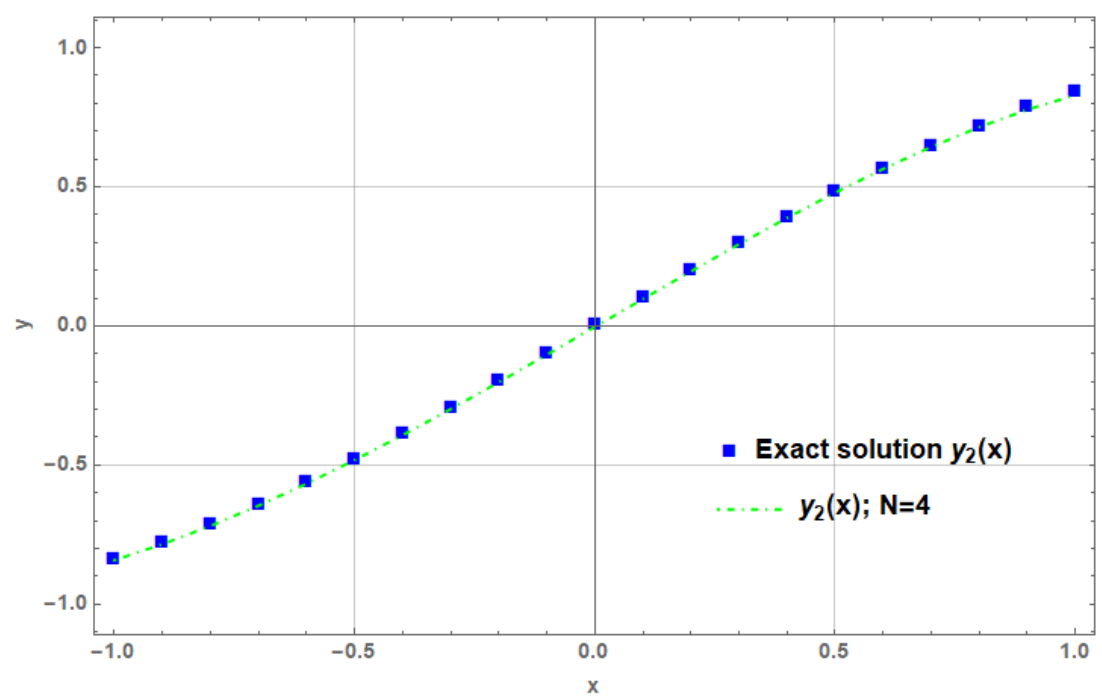

(b) Exact solution and Charlier series solution $y_{2}(x)$ for $\alpha=0.1$

Figure 2. Displacements of the solutions for Example 4.2

Table 3. Comparison of CPU timing and the absolute errors with respect to the Charlier series solutions $\left\{y_{1}(x), y_{2}(x)\right\}$ with $\alpha=0.1$ and $N$ for Example 4.2

\begin{tabular}{rllll}
\hline \multicolumn{1}{c}{$x_{i}$} & $\left|e_{1}\left(x_{i}\right)\right|$ & $\left|e_{2}\left(x_{i}\right)\right|$ & $\left|e_{1}\left(x_{i}\right)\right|$ & $\left|e_{2}\left(x_{i}\right)\right|$ \\
& $N=4$ & $N=4$ & $N=10$ & $N=10$ \\
\hline-0.6 & $2.9193 \mathrm{e}-03$ & $1.6008 \mathrm{e}-03$ & $3.5522 \mathrm{e}-010$ & $3.5888 \mathrm{e}-011$ \\
-0.2 & $2.6760 \mathrm{e}-03$ & $2.9928 \mathrm{e}-03$ & $4.3578 \mathrm{e}-010$ & $1.0054 \mathrm{e}-010$ \\
0.2 & $7.2685 \mathrm{e}-04$ & $1.9825 \mathrm{e}-03$ & $5.3109 \mathrm{e}-010$ & $1.0237 \mathrm{e}-010$ \\
0.6 & $1.9825 \mathrm{e}-03$ & $1.1841 \mathrm{e}-04$ & $4.6041 \mathrm{e}-010$ & $1.9166 \mathrm{e}-010$ \\
1.0 & $1.0676 \mathrm{e}-03$ & $1.1410 \mathrm{e}-02$ & $6.7767 \mathrm{e}-010$ & $5.9516 \mathrm{e}-009$ \\
\hline Time & & 0.0469 & & 0.4063 \\
\hline
\end{tabular}

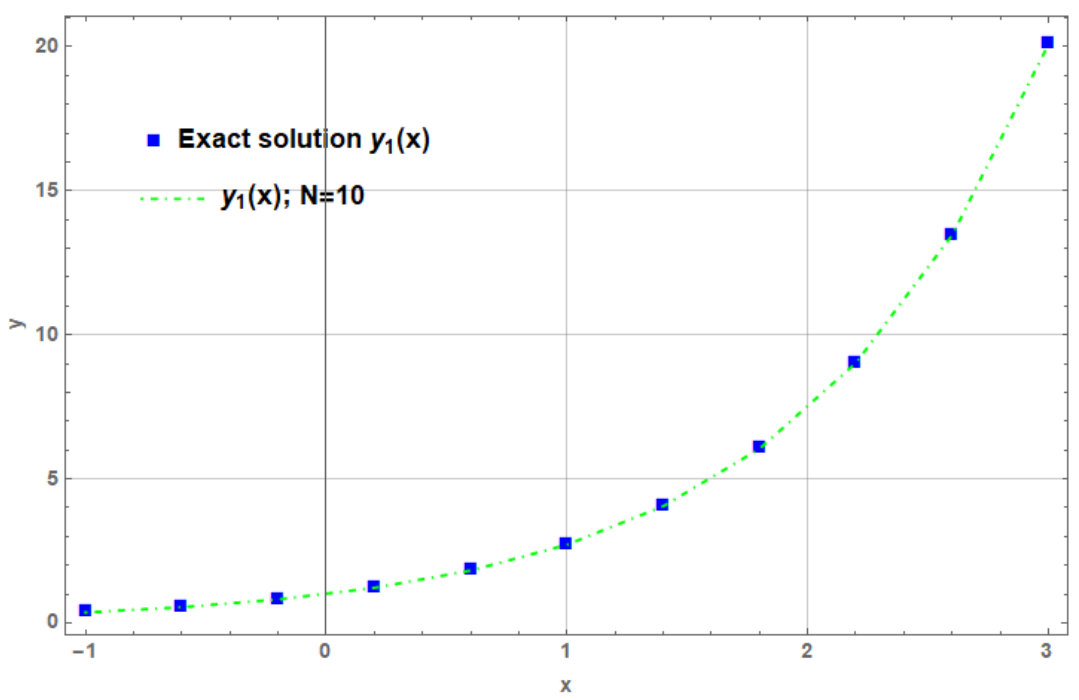

(a) Exact solution and Charlier series solution $y_{1}(x)$ for $\alpha=0.1$. 


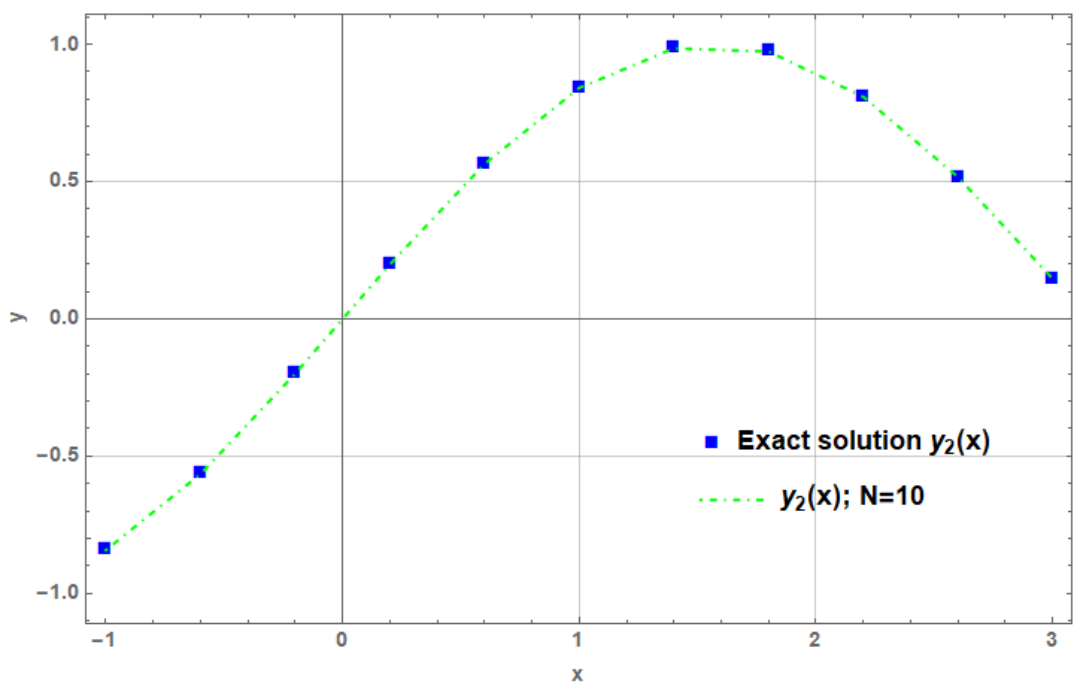

(b) Exact solution and Charlier series solution $y_{2}(x)$ for $\alpha=0.1$.

Figure 3. Displacements of the solutions on [-1,3] for Example 4.2.

\section{Conclusions}

SDDEs have been suitably solved by the present method based on the Charlier polynomials and the collaboration of the matrices of the terms in the system (1). In that case, a computer program of the method has managed to perform its routines on a considered example. Thus, the numerical and graphical investigations have revealed the applicability and practicability of the present method, as can be seen in Figures 1-3 and Tables 1-3. Although SDDEs have the complicated terms in virtue of the delay arguments, the present method solves two examples immediately, as noticed in Tables 1 and 3. In addition, Tables 1 and 2 show that the parameter$\alpha$ has played a key role in the accuracy for its different values. The present method sheds light on new aspect and advancement regarding the use of the combinatorial polynomials in SDDEs. Thereby, one can admit that the present method is inventive and straightforward to employ the systems of integro-differential-delay and nonlinear differential-delay equations, of course, some modifications can be implemented.

\section{Author Statement}

The authors confirm contribution to the paper as follows: study conception and design: M. Sezer, Ö.K. Kürkçü; data collection: Ö.K. Kürkçü; analysis and interpretation of results: M. Sezer, Ö.K. Kürkçü; draft manuscript preparation: Ö.K. Kürkçü. All authors reviewed the results and approved the final version of the manuscript.

\section{Conflict of Interest}

The authors declare no conflict of interest.

\section{References}

Abdel-Halim Hassan, I.H. (2008). Application to differential transformation method for solving systems of differential equations. Applied Mathematical Modelling, 32(12), 25522559. 
Akyüz, A., \& Sezer, M. (2003). Chebyshev polynomial solutions of systems of high-order linear differential equations with variable coefficients. Applied Mathematics and Computation, 144, 237-247.

Butzer, P.L., Hauss, M., \& Schmidt, M. (1989). Factorial functions and Stirling numbers of fractional orders. Results in Mathematics, 16, 16-48.

Culshaw, R.V., \& Ruan, S. (2000). A delay-differential equation model of HIV infection of CD4 ${ }^{+}$ T-cells. Mathematical Biosciences, 165(1), 27-39.

Cushing, J.M. (1977). Integro differential Equations and Delay Models in Population Dynamics. Heidelberg: Springer-Verlag.

Deniz, S. (2021). Semi-analytical approach for solving a model for HIV infection of CD4+ Tcells. TWMS Journal of Applied and Engineering Mathematics, 11(1), 273-281.

Dunster, T.M. (2001). Uniform asymptotic expansions for Charlier polynomials. Journal of Approximation Theory, 112, 93-133.

Gokmen, E., Isik, O.R., \& Sezer, M. (2015). Taylor collocation approach for delayed LotkaVolterra predator-prey system. Applied Mathematics and Computation, 268, 671-684.

Gökmen, E., \& Sezer, M. (2013). Taylor collocation method for systems of high-order linear differential-difference equations with variable coefficients. Ain Shams Engineering Journal, 4, 117-125.

Huang, X.-M., Lin, Y., \& Zhao, Y.-Q. (2021). Asymptotics of the Charlier polynomials via difference equation methods. Analysis and Applications, 19(04), 679-713.

Kuang, Y. (1993). Delay Differential Equations with Applications in Population Dynamics. New York: Academic Press.

Pamuk, S. (2005). The decomposition method for continuous population models for single and interacting species. Applied Mathematics and Computation, 163, 79-88.

Roman, S. (1984). The umbral calculus. New York: Academic Press.

Srivastava, H.M., \& Deniz, S. (2021). A new modified semi-analytical technique for a fractionalorder Ebola virus disease model. RACSAM 115, 137. DOI:10.1007/s13398-021-01081-9.

Szegö, G. (1975). Orthogonal polynomials. Providence Rhode Island: American Mathematical Society.

Tatari, M., \& Dehghan, M. (2009). Improvement of He's variational iteration method for solving systems of differential equations. Computers $\mathcal{E}$ Mathematics with Applications, 58(11-12), 2160-2166.

Thirumalai, S., Seshadri, R., \& Yuzbasi, S. (2021). Spectral solutions of fractional differential equations modelling combined drug therapy for HIV infection. Chaos, Solitons and Fractals, 151, 111234.

Varma, S., \& Taşdelen, F. (2012). Szász type operators involving Charlier polynomials. Mathematical and Computer Modelling, 56, 118-122.

Yüzbaşı, Ş., \& Karaçayır, M. (2017). An exponential Galerkin method for solutions of HIV infection model of CD 4+ T-cells. Computational Biology and Chemistry, 67, 205-212. 